\title{
LA-UR $90-2968$
}

DE9 I 000444

TITLE: Modeling of the Quasi-Steady Magnetotail

AUTHOR(S): J. Birn and K. Schindler

SUBmitTED TO: Proceedings of the Second Huntsville Workshop on

Magnetosphere-Ionosphere Plasma Models, 11-13 October 1989

in Huntsville, $\mathrm{AL}$

\section{DISCLAIMER}

This report was prepared as an account of work sponsored by an agency of the United States Government. Neither the United States Government nor any agency thereof, nor any of their employees, makes any warranty, express or implied, or assumes any legal liability or responsibility for the accuracy, completeness, or usefulness of any information, apparatus, product, or process discloset, or represents that its use would not infringe privately owned rights. Reference herein to any specific commercial product, process, or service by trade nanre, trademark, manufacturer, or otherwise does not necessarily constitute or imply its endorsement, recommendation, or favoring by the United States Government or any agency thereof. The views and opinions of authors expressed herein do not necessarily state cir reflect those of the United States Government or any agency thereof.

By acceptance of this article, the publisher recognizes that the US Government retains a nonexclusive. royalty-free license to publish or reproduce the publisned form of this contribution, or to allow others to do so. for U.S. Government purposes

The Los Alamos National Laboratory requests that the publisher identify this article as work performed under the auspices of the U.S. Department of Energy
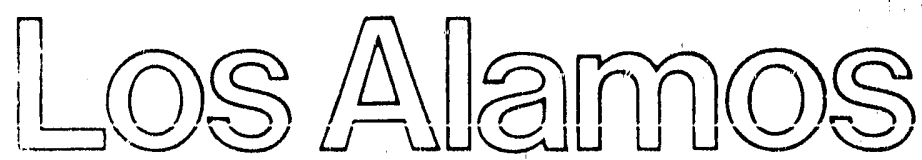


\title{
Modeling of the Quasi-Steady Magnetotail
}

\author{
Joachim Birn \\ University of California, Los Alamos National Laboratory, Los Alamos, NM 87545
}

Karl Schindler

Ruhr-Universität, D4630 Bochum 1, W. Germany

Abstract. The three-dimensional theory of the quiet magnetotail is reviewed and updated. The most advanced formulation of the theory allows one to solve the magnetohydrodynamic (MHD) equations including field-aligned flow by reduction to a set of ordinary differential equations and an ordinary integral, These solutions represent lowest order solutions of an asymptotic expansion of the MHD equations for small electric field and weak time dependence. Applications to the magnetotail configuration are presented, which include the following properties in a self-consistent way: Flaring of the tail in $y$ and $z$, associated with the presence of $B_{y}$ and $B_{z}$; variation of plasma sheet and current sheet thickness with $x$ and $y$, associated with variations of $B_{z}$; field-aligned currents at the boundary between plasma sheet and lobes with the signature of "region 1" currents, i.e., toward the Earth on the dawn side and away on the dusk side, associated with the decrease of the tail flaring with distance from the Earth; net cross-tail magnetic field. Particular steady models with field-aligned flow demonstrate the important role of the flow in allowing or producing configurations with a transition from a thick closed plasma sheet to a much thinner distant open current sheet and for the possibility of thick plasmoids propagating in near equilibrium through the thin distant current sheet. 


\section{INTRODUCTION}

The purpose of this paper is to present our most advanced description of the quasi-steady magnetotail structure. It is based largely on a summary and update of recent work of the authors [Schindler and Birn, 1986; Birn, 1987, 1989, 1990a,b] on magnetotail theory and modeling. A magnetospheric model is usually considered as an exact or approximate solution of the time-independent or weakly time-dependent magnetohydrodynamic (MIID) equations, which can be represented in the following form, using common notation and MKS units:

$$
\begin{aligned}
\partial \rho / \partial t & =-\nabla \cdot \rho \mathbf{v}, \\
\partial \rho \mathbf{v} / \partial t & =-\nabla p-\nabla \cdot(\rho \mathbf{v v})+\mathbf{j} \times \mathbf{B}, \\
\mathbf{E}+\mathbf{v} \times \mathbf{B} & =0 \\
\partial\left(p / \rho^{\gamma}\right) / \partial t & =-\mathbf{v} \cdot \nabla\left(p / \rho^{\gamma}\right), \\
\nabla \cdot \mathbf{B} & =0 \\
\nabla \times \mathbf{B} & =\mu_{o} \mathbf{j}, \\
\partial \mathbf{B} / \partial t & =-\nabla \times \mathbf{E}
\end{aligned}
$$

The most important assumption included in (1)-(7) is that of isotropic particle distribution functions (in the frame of the bulk flow), which implies a scalar pressure as well as an adiabatic convection law (4), following from the absence of heat flux. For three-dimensional isotropic distributions the adiabatic index $\gamma$ should equal 5/3. In addition, collisions are neglected, except for their possible implicit role in isotropizing the pressure.

Usually, for the description of quiet magnetosphere structures, the inertia terms in (2) are neglected, which leads to the quasi-static force balance

$$
\nabla p=\mathbf{j} \times \mathbf{B}
$$

This approximation is valid for flow speeds that are small in comparison to typical Alfvén wave or sound speeds. In this case, Equations (8), (5), and (6) represent the magnetostatic equations, which remain from (1)-(7) for $\partial / \partial t=0, \mathbf{v}=\mathbf{0}$, and $\mathbf{E}=\mathbf{0}$. 
Three-dimensional solutions of (1)-(7), or just of the magnetostatic subset; are not available yet for the entire magnetosphere. Models including the near-Earth magnetosphere inside of $10-20 R_{E}$ are still mostly empirical [e.g., Tsyganenko, 1987, 1989], fulfiling (5) and (6) by construction, but satisfying the force balance (2) or (8) only to the extent that the assumed magnetic field expressions and the data set used for determination of free parameters in the field expressions are consistent with it [see, e.g., Spence et al., 1987]. For the magnetotail, however, fairly general solutions of (1)-(7) can be derived [e.g., Birn, 1987, $1990 \mathrm{~b}\}$, due to the fact that the characteristic length scale $L_{z}$ for variations in the $z$ direction perpendicular to the plasma sheet is much smaller than the length scales in the other space directions, except for regions in the vicinity of the magnetopause. In the following section we will present the most recent advances in solving (1)-( 7$)$, which provide now a method to reduce these equations to a set of ordinary differential equations and an ordinary integral in the case of $\partial / \partial t=0$ and $\mathbf{E}=0$, when the flow is along the magnetic field in a certain frame of reference [Birn; 1990b] or vanishes completely [Birn, 1987]. Particular solutions, illustrating typical properties of the self-consistent models, will be presented in sections 3 and 4 . Solutions for $\mathrm{E} \neq 0$ and $\partial / \partial t \neq 0$, which can be gained for the quasistatic case, will be discussed in section 5 .

\section{GENERAL SOLUTION FOR $\mathbf{E}=0$ AND $\partial / \partial t=0$}

For $\mathrm{E}=0$, Equation (3) implies that the flow velocity is parallel to the magnetic field, i.e.

$$
\mathbf{v}=m \mathbf{B} / \sqrt{\mu_{o} \rho}
$$

where $m$ denotes the Alfvén Mach number. We will represent the magnetic field B by Euler potentials $\alpha$ and $\beta$, i.e.,

$$
\mathrm{B}=\nabla \alpha \times \nabla \beta
$$

which solves Eq. (5). Equation (1) with $\partial / \partial t=0$ now yields

$$
\mathbf{B} \cdot \nabla(m \sqrt{\rho})=0,
$$

or

$$
m \sqrt{\rho}=f(\alpha, \beta)
$$

where $f$ is a quantity that is constant on field lines and thus a yet unspecified function of $\alpha$ and $\beta$. In a similar way, we find from Equation (4)

$$
p / \rho^{\gamma}=g(\alpha, \beta)
$$


Equations (11) and (12) can be combined to

$$
p m^{2 \gamma}=F(\alpha, \beta)
$$

where

$$
F(\alpha, \beta)=f^{2 \gamma} g
$$

Using Eqs. (6) and (9) to replace $\mathbf{j}$ and $\mathbf{v}$ in (2), we find

$$
\nabla\left(p+\frac{1}{2 \mu_{0}} B^{2}\right)=\frac{1}{\mu_{0}} \cdot \nabla \cdot\left\{\left(1-m^{2}\right) \mathbf{B B}\right\} .
$$

Our basic set of equations has now been reduced to (15) together with (1.3) and (5) or (10), which are to be solved for $p, m$, and $\mathbf{B}$. The density $\rho$ then follows from (11) or (12), and the velocity $\mathbf{v}$ from (10).

The further solution of Eq. (15) with (13) and (5) is based on the assumption that the configuration varies predominantly in the $z$ direction with weak variations in the other two space directions. Using a smallness parameter $\epsilon$ to describe the weak variation in $x$ and $y$, we can find the scaling

$$
\partial / \partial z, B_{x}, B_{y}=O(1), \quad \partial / \partial x, \partial / \partial y, B_{z}=O(\epsilon)
$$

with $p$ and $m$ being also of order unity. The scaling of $B_{z}$ in (16) is found from consistency with Eq. (5). A possible additional constant contribution to $B_{z}$ of arbitrary magnitude is chosen to vanish, consistent with the magnetotail properties. The asymptotic theory allows $B_{y}$ to be of order unity, although the average $B_{y}$ in the magnetotail is about as small as $B_{z}$.

We introduce further the total pressure $\hat{p}$ by

$$
\hat{p}=p+\frac{1}{2 \mu_{o}} B^{2}
$$

From Eq. (15), we now find that

$$
\frac{\partial \hat{p}}{\partial z}=O\left(\epsilon^{2}\right)
$$

Neglecting $\epsilon^{2}$ compared to unity, we obtain the frequently used pressure balance equation

$$
p+\frac{1}{2 \mu_{\circ}} B^{2}=\hat{p}(x, y)
$$

which has thus not been altered by the presence of the parallel flow. We rewrite the other two components of (15) introducing the new variable $\tau$ by

$$
\tau=\int d s / B
$$


where the integral extends along fleld lines from an arbitrary lower boundary. Using this variable, which represents the yolume of a magnetic flux tube of unit flux, we can write

$$
\frac{d h}{d \tau} \equiv \mathbf{B} \cdot \nabla h
$$

where $h$ is an arbitrary quantity, and $d / d \tau$ denotes the derivative along a field line, i.e., for constant $\alpha$ and $\beta$

$$
\frac{d h}{d \tau} \equiv \frac{\partial h(\alpha, \beta, \tau)}{\partial \tau}
$$

Equation (15) with (5) now yields

$$
\begin{aligned}
& \left(1-m^{2}\right) \frac{d B_{x}}{d \tau}-B_{x} \frac{d m^{2}}{d \tau}=\mu_{0} \frac{\partial \hat{p}}{\partial x} \\
& \left(1-m^{2}\right) \frac{d B_{y}}{\dot{i} \tau}-B_{y} \frac{d m^{2}}{d \tau}=\mu_{0} \frac{\partial \hat{p}}{\partial y}
\end{aligned}
$$

From (14) and (19), we find

$$
\begin{gathered}
\frac{d m^{2}}{d \tau}=-\frac{1}{\gamma} \frac{m^{2}}{p} \frac{d p}{d \tau} \\
\frac{d p}{d \tau}=B_{x} \frac{\partial \hat{p}}{\partial x}+B_{y} \frac{\partial \hat{p}}{\partial y}-\frac{1}{\mu_{o}}\left(B_{x} \frac{d B_{x}}{d \tau}+B_{y} \frac{d B_{y}}{d \tau}\right) .
\end{gathered}
$$

If (26), (23), and (24) are inserted into (25) and $p$ is eliminated using (13), we obtain

$$
\frac{d m^{2}}{d \tau}=\frac{\mu_{o} \sigma^{2} m^{2}}{1-m^{2}-\sigma^{2}\left(B_{x}^{2}+B_{y}^{2}\right)}\left(B_{x} \frac{\partial \hat{p}}{\partial x}+B_{y} \frac{\partial \hat{p}}{\partial y}\right)
$$

where

$$
\sigma^{2}=\frac{1}{\mu_{o} \gamma} \frac{m^{2}}{p}=\frac{m^{2+2 \gamma}}{\mu_{o} \gamma F}
$$

with

$$
\begin{aligned}
& \frac{d x}{d \tau} \equiv \mathbf{B} \cdot \nabla x=B_{x}, \\
& \frac{d y}{d \tau} \equiv \mathbf{B} \cdot \nabla y=B_{y}
\end{aligned}
$$

We now have a complete set of explicit first order differential equations for $x, y, B_{x}, B_{y}$, and $m^{2}$, given by (23), (24), (27), (29), and (30) with an arbitrary choice of $\hat{p}(x, y)$ and $F(\alpha, \beta)$. This set can be integrated by standard numerical procedures. The solution follows in the form $x(\tau ; \alpha, \beta), y(\tau ; \alpha, \beta)$, which gives $\beta=\beta(x, y, \alpha)$ if $\tau$ is eliminated. 
For further integration, we use the representation of $\mathbf{B}$ by Euler potentials, Eq. (10), and obtain

$$
B_{x}^{2}+B_{y}^{2}=|\nabla \beta|_{\alpha}^{2}\left(\frac{\partial \alpha}{\partial z}\right)^{2}
$$

where

$$
|\nabla \beta|_{\alpha}^{2}=\left(\frac{\partial \beta(x, y, \alpha)}{\partial x}\right)^{2}+\left(\frac{\partial \beta(x, y, \alpha)}{\partial y}\right)^{2}
$$

Solving the pressure balance (19) for $\partial \alpha / \partial z$, we then find $z$ as a function of $x, y$, and $\alpha$ by integration:

$$
z= \pm \int|\nabla \beta|_{\alpha} \frac{d \alpha}{\sqrt{2 \mu_{o}(\hat{p}-p)}}
$$

In (33), $p$ is a function of $x, y$, and $\alpha$, given by (13), with $m^{2}$ and $\beta$ expressed as functions of $x, y$, and $\alpha$ through the solutions of our set of ordinary differential equations (23), (24), (27), (29), and (30).

The number of differential equations can be reduced using an integral which can be found from (23), $(24)$, and (27):

$$
\frac{\gamma}{\gamma-1} m^{2(1-\gamma)}+m^{2(2-\gamma)}-m^{4} \hat{p} / F=R(\alpha, \beta) \text { for } \gamma \neq 1
$$

and

$$
m^{2}+\ln m^{2}-m^{4} \hat{p} / F=R(\alpha, \beta) \quad \text { for } \gamma=1 \text {. }
$$

(A further reduction can be obtained, if the variable $\tau$ is replaced, for instance, by $x$ through (29).)

In the static case, $\mathbf{v}=\mathbf{0}$ or $m=0$, one finds the simplified set of equations:

$$
\begin{aligned}
& d B_{x} / d \tau=\mu_{0} \partial \hat{p} / \partial x \\
& d B_{y} / d \tau=\mu_{0} \partial \hat{p} / \partial y
\end{aligned}
$$

together with (29), (30), and (33), where $p$ is now a function of $\alpha$ and $\beta$, to be determined from boundary conditions or other constraints.

\section{SPECIAL STATIC SOLUTIONS}

$3: 1 \hat{p}=\hat{p}(r)$ where $r^{2}=(x-a)^{2}+(y-b)^{2}$

It is particularly easy to derive static solutions. when the total pressure function can be written as a function of $r$ only in a cylindrical coordinate system $r, \phi, z$ with an arbitrary center at $x=a, y=b[$ Birn, 
1979, 1987]. In this case one finc's $B \cdot \nabla\left(r B_{\phi}\right)=0$, i.e., $r B_{\phi}$ is constant along any fleld line. For the average magnetotail configuration it is reasonable to assume symmetry around the equatorial plane. This implies

$$
B_{\phi} \equiv 0
$$

that is, field lines must lie in planes $\phi=$ const. In this case, the coordinate $\phi$ can be chosen as one of the Euler potentials, say,

$$
\beta=\phi
$$

For suitable choices of $p=P(\alpha, \beta)$, the final solution can be given in analytic form. For instance, for

$$
p=p_{N} \exp (-2 \lambda(\beta) \alpha)
$$

the magnetic field and the pressure are given by [Birn, 1987]

$$
\begin{gathered}
B_{r}=-\hat{B} \tanh \zeta \\
B_{z}=\frac{z}{r} \frac{d}{d r}(r \hat{B}) \tanh \zeta-L \frac{d \hat{B}}{d r} \\
p=\hat{p}(r) / \cosh ^{2} \zeta
\end{gathered}
$$

where

$$
\begin{gathered}
\hat{B}(r)=\sqrt{2 \mu_{0} \hat{p}(r)}, \\
L(r, \phi)=[\lambda(\phi) r \hat{B}(r)]^{-1},
\end{gathered}
$$

and

$$
\zeta=z / L(r, \phi)
$$

The solution (40)-(45) still contains two free functions, $\hat{B}(r)$, the lobe magnetic field strength, and $\lambda(\phi)$, a function that determines the variation of tine characteristic scale length $L$, equivalent to a current sheet half-thickness, across the tail. Both functions can be reasonably well adjusted to observations. A particular model is shown in Figure 1. It is adjusted to tail observations between 20 and $60 R_{E}$ and extended to $140 R_{E}$. The figure shows magnetic field lines in three different planes $\phi=$ const with the dashed line indicating the scale length $L$ and the shaded region representing the region of closed (that is, plasma sheet) field lines. This particular example does not include a distant neutral line to terminate the closed field line region. With a different choice for $\hat{B}(r)$, however, a distant neitral line can also be modeled. 
Despite the restacting assumptions used for the derivation of $(40)-(45)$; this solution includes several properties necessary for a quantitatively satisfying magnetotail model: A flaring of the tail with both $y$ and $z$, which can be adjusted; the presence of a normal field component $B_{z}$ in the neutral sheet with the balance of the corresponding Loren: forces by pressure gradients; a variation of the current sheet and plasma sheet thickness along and across the tail, associated with a variation of $B_{z}$, which can also be adjusted. The major restriction is the absence of field-aligned curents, which follows from the assumed form for $\hat{p}$ and the consequence that field lines lie in planes $\phi=$ const. This condition will be released in the following subsection.

\subsection{Extended tail solutions with $j_{\|} \neq 0$}

For more general functions $\hat{p}(x, y)$ analytic solutions seem no longer possible, and Equations (35), (36), (29), (30), and (33) have to be integrated numerically. A particular solution, obtained by Birn [1989], is shown in Figure 2. The figure shows, in a perspective view from the tail, magnetic flux surfaces formed by field lines that originate from a circle of $20 R_{E}$ radius at $x=-20 R_{E}$ out to $x=-120 R_{E}$. If the magnetoparse were a flux surface (that is, a tangential discontinuity) and if the present model would be valid out to the magnetopause and if we assume that the magnetopause is indeed a circle of $20 R_{E}$ radius at $x=-20 R_{E}$, then this surface would in fact represent the magnetopause shape of the model. The most prominent feature of this particular solution, representative of others, is the bulging of the tail in the low latitude plasma sheet region. This bulging is due to a difference in the degree of flaring of field lines in their equatorial projections between plasma sheet and lobes, corresponding to a shear of the magnetic field, that is, a rotation of the equatorial projection of $\mathrm{B}$ with altitude $z$ above the equatorial plane. It is thus directly related to the presence of field-aligned currents which produce such a shear. In the present $m$ inl, it is the consequence of just two boundary conditions, namely an adjustment of the tail flaring in the near tail between 20 and $60 R_{E}$ to the observed flaring and the requirement that at large distances field lines should become aligned with the tail axis, which implies a decrease of the flaring with distance, as is also observed [Slavin et al., 1985].

The distribution of the field-aligned currents at $x=-20 R_{E}$ is shown more explicitly in Figure 3 . The top part of the figure shows lines of constant current density $j_{\|}$in a north-dusk sector of the tail, while the bottom part shows the profile of $j_{\|}$as a function of $z$ for constant $x$ and $y$. The current densities are normalized by $j_{N}$, the peak cross-tail current density at $x=-20 R_{E}$ and $y=0$. The contour lines in 
the top panels represent $j_{\|}$values at multiples of $2.5 \%$ of $j_{N}$. The signature of these currents is that of region 1 field-aligned currents flowing toward the Earth on the dawnside and away on the duskside [Iijima and Potemra, 1976]. The total current $I_{\|}$in each direction is about $4 \times 10^{5} \mathrm{~A}$ using a lobe field strength $B_{N}=25 \mathrm{nT}$ at $x=-20 R_{E}$. The magnitude of $I_{\|}$increases toward the Earth. This indicates that not only contributions from further downtall are conserved, but that new contributions from the near flanks are added closer to the Earth. We do, therefore, expect that these field-aligned currents actually reach the Earth and very likely become even larger due to additional contributions from the morning and evening flanks not included in our model. These values therefore seem consistent with observations of the region 1 currents that persist even during quiet times [Iiiima and Potemra, 1976].

\subsection{Solutions with net cross-tail magnetic field}

The symmetry of the magnetotail models of sections 3.1 and 3.2 with respect to the midnight meridian and the equatorial planes is a reasonable assumption for the average magnetotail. "or a particular instantaneous model, however, the absence of a net cross-tail magnetic field component $B_{y N}$, which is $T^{\prime:-}$ quired for such symmetry, is a rather singular case [e.g., Fairfield, 1979; Lui, 1984; Tsurutani et al., 1984]. Inclusion of the asymmetry associated with the net $B_{y N}$ requires, in general, again numerical integration of Equations (35), (36), (29), (30), and (33). Such solutions were obtained by Birn [1990a]. Figure 4 shows a representative example, again in the form of a magnetic flux surface originating from $r=20 R_{E}$ at $x=-20 R_{E}$, drawn in a perspective view from the tall. The magnitude of the net cross-tail field component $B_{y N}$ at $x=-20 R_{E}$ is $2 \%$ of the lobe field strength, which is rather typical [Fairfield, 1979]. Note that, in contrast to two-dimensional $(\partial / \partial y=0)$ models [e.g., Voigt and Hilmer, 1987], $B_{y}$ is no longer constant along field lines in a three-dimensional model.

Figure 4 demonstrates two new characteristic effects. The first one is a separation of the two lobe regions from each other, increasing with distance down the tail. This can also be found in the simpler two-dimensional models [e.g., Hilmer and Voigt, 1987]. It is a direct consequence of the fact that the net $B_{y}$ field persists through the lobes. Whether this effect corresponds to a similar behavior of the magnetopause, depends on to what extent the magnetopause is open in these regions. If we postulate a symmetric magnetopause surface, Figure 4 suggests an asymmetric opening of such magnetopause, on the north-dawn and south-disk sides for our present choice of positive $B_{y N}$. Such an asymmetric opening is indeed supported by observations of $B_{y}$ related asymmetries in the presence of magnetosheath plasma in 
the lobes [Hardy et al., 1976; Gosling et al., 1985].

The second effect demonstrated by Figure 4 is a tilt of the closed plasma sheet region, indicated by shading, against the neutral sheet (defined by $B_{x}=0$ ), which, by assumption, is identical with the equatorial plane $z=0$. This effect is caused by field lines which have one end at $x=-20 R_{E}$ inside the circle $r=20 R_{E}$, cross the equatorial plane, but do not return to within the $20 R_{E}$ circle at $x=-20 R_{E}$, but rather extend to larger distances in $|y|$. These field lines are likely to be interconnected with the magnetosheath field through the low latitude boundary region at their other end, so that they become topologically similar to the lobe field lines. A quantitative estimate of this effect is provided by Figure 5 , which shows the boundary of the closed field line region in the equatorial plane for various magnitudes of the net $B_{y N}$, indicated by the parameter $b_{N}$ (representing $B_{y N}$ normalized by a characteristic lobe field strength). The dashed line represents a distant neutral line or separator, which terminates the closed field line region for $b_{N}=0$. For increasing $b_{N}$ the region of closed field lines shrinks, despite the fact that $B_{z}$ remains positive everywhere earthward of the dashed line. While for $b_{N}=0.027$ (corresponding to $2.7 \%$ of the typical lobe field), which is typical for the average net $B_{y}$ field [Fairfield, 1979], the effect is not very strong, it becomes appreciable for $b_{N}=0.107$, which is not extremely unusual.

The possible existence of field lines which cross the neutral sheet with positive $B_{z}$, but are nevertheless open at one end, is of interest for the interpretation of observed properties in the vicinity of the low latitude magnetopause. Depending on the recent history of such field lines and on the location where they are interconnected with the magnetosheath, one expects different plasma properties. If the field lines have become interconnected with the magnetosheath quite recently, one would expect to see a mixture of magnetospheric and magnetosheath plasma, as typically observed in the low latitude boundary layer [e.g., Eastman and Hones, 1979]. If they have been interconnected for quite a while, one would expect to see only magnetosheath like plasma, as is typical for the plasma mantle [Rosenbauer et al., 1975], or no plasma at all, if the interconnection location is far away. Such density drops inside the low-latitude magnetopause are indeed observed [Gosling et al., 1986]. In any case, the suggested interpretation would require a strong dependance of the occurrence of the observed phenomenon on the magnitude of the net $B_{y}$ (or the interplanetary $B_{y}$ ). This will allow one to find out from statistical data studies whether the presence of such type of open field lines indeed plays a role in influencing the low-latitude boundary properties. 


\section{STEADY FLOW SOLUTIONS}

The quasi-static approximation, neglecting the inertia effects in the force balance, is usually a good assumption for the closed plasma sheet region during quiet times. This approximation, however, seems no longer valld in the more distant tail near and beyond the termination of the closed field line region, where fast flows seem to prevail most of the time [Zwickl et al., 1984]. Since these flows are typically strongly fleld-aligned, it seems justified to apply the theory presented in section 2. One possible application is the description of the termination region itself. Further posssible applications of that theory include the motion of a plasmoid through the distant magnetotail [observed, e.g, by Hones et al., 1984], which in the framt of the moving plasmoid may be considered as the steady flow of a plasma around it, and magnetopause structures, which are often found to have an associated "de Hoffmann-Teller" frame, in which the electric field vanishes [e.g., Papamastorakis et al., 1989; Sonnerup et al., 1990].

A particular example, representing the steady motion of a plasmoid through the distant tail current sheet, was derived by Birn [1990b], using an incompressible two-dimensional model. This example is represented in' Figure 6, showing magnetic fleld lines, which are also the flow lines in the frame moving with the plasmoid. In this frame, the plasma inside the plasmoid is at rest. This is a consequence of the symmetry around $z=0$, which implies that $m=0$ at $z=0$ and, via Equation (11), $m=0$ along each closed plasmoid field line. The three panels of Figure 6 correspond to three different parameter sets with the top panel representing the static case without flow, while the two bottom panels correspond to fast, nearly Alfvénic flow ( $m=0.995$ on open field lines around the plasmoid). The first two panels correspond to $20 \%$ enhancement of the total pressure $\hat{p}$ at the center of the plasmoid as compared to $\hat{p}$ at large distances, while the last panel corresponds to $70 \%$ pressure enhancement. The $x$ coordinate is normalized by an arbitrary length $L_{x}$ in each case. The $z$ coordinate on the left hand side of each panel is scaled by a length $L_{c}$, which represents the current sheet half-width far away from the plasmoid (large $\left.|x|\right)$ in the absence of flow. The vertical scales on the right hand sides are normalized by the actual current sheet half-width $L$ for $|x| \rightarrow \infty$ in the presence of the flow. These scales indicate the characteristic difference in the island thickness between the static case (top panel) and the two fast flow cases (two bottom panels). In the static case, the island width is comparable to the current sheet scale $L$, while the islands are much thicker for the two fast flow cases. Observations indicate that the ambient current layer or plasma sheet in the far tail is much thinner than the plasmoid. This follows, for instance, from the fact that the plasmoid is typically 
entered from the tail lobes [see, e.g., Baker et al., 1987]. In the present model, this requires values of $m$ close to 1 if the pressurt enhancement in the center of the plasmoid is only moderate. Observations of magnetic pressure enhancement outside the plasmoid, which should be equivalent to the enhancement of the total pressure, show typical increases of $20-70 \%$ [Slavin et al., 1984]. It can easily be shown that much larger pressure enhancements are necessary to produce a thick island in the static case. These results thus demonstrate the necessity of including inertia effects in a realistic description of plasmoids in the distant magnetotail.

Although the model of Figure 6 was constructed to illustrate plasmoid properties, it may also be used to illustrate possible properties of the quasi-steady magnetotail near the termination of the closed field line region. This region is found observationally as the transition region from slow or no flow in the thick closed plasma sheet to fast flow in the typically much thinner more distant current sheet and the adjacent lobes [Zwickl et al., 1984]. If we consider only the right half or less of the lower two panels in Figure 6, these sections may be similar to the plasma sheet termination region. There is no flow in the closed field line region, but fast flow in the regions surrounding it and extending down the tail. Again we can conclude that the transition from a thick closed field line region to a thin current sheet further down the tail should be associated with fast plasma flow.

\section{SOLUTIONS FOR $\mathbf{E} \neq 0$ AND $\partial / \partial t \neq 0$}

For the quasi-static case, general solutions for $\mathbf{v}$ and $\mathbf{E}$ can be derived for any (weakly) time-dependent sequence of equilibria $\mathbf{B}(\mathbf{r}, t)$ and $p(\mathbf{r}, t)$, if boundary conditions are imposed only at one point on each magnetic field line [Schindler and Birn, 1978]. Using an Euler representation $\alpha(\mathbf{r}, t), \beta(\mathbf{r}, t)$ for the magnetic field, the electric field can be r'presented by

$$
\mathbf{E}=\frac{\partial \beta}{\partial t} \nabla \alpha-\frac{\partial \alpha}{\partial t} \nabla \beta-\nabla \Phi(\alpha, \beta, t)
$$

where $\Phi(\alpha, \beta, t)$ is arbitrary and has to be obtained from boundary conditions or other appropriate assumptiors. The velocity perpendicular to $\mathbf{B}$ is given by the $\mathbf{E} \times \mathbf{B}$ drift:

$$
\mathrm{v}_{\perp}=\mathrm{E} \times \mathrm{B} / B^{2}
$$

The parallel component of the flow velocity can be determined from the adiabatic law (4) and the continuity equation (1), which can be combined to

$$
\mathrm{D} p / \mathrm{D} t \equiv \partial p / \partial t+\mathbf{v} \cdot \nabla p=-\gamma p \nabla \cdot \mathbf{v}
$$


Using our curvilinear coordinate system $\alpha, \beta$, and $\tau$, and splitting the velocity into its parallel and perpendicular components, we can express (52) by

$$
\frac{d}{d \tau}\left(\frac{v_{\|}}{B}\right)+\nabla \cdot \mathbf{v}_{\perp}=-\frac{1}{\gamma p} \frac{\mathrm{D} p}{\mathrm{D} t}
$$

where $d / d \tau$ again denotes the derivative along a field line, i.e., for constant $\alpha$ and $\beta$. Equation (53) can be integrated along the field lines:

$$
\frac{v_{\|}}{B}=\left.\frac{v_{\|}}{B}\right|_{0}-\frac{\tau}{\gamma p} \frac{\mathrm{D} p}{\mathrm{D} t}-\int \nabla \cdot \mathbf{v}_{\perp} d \tau
$$

This equation can also be expressed in the following form [Schindler and Birn, 1978]:

$$
\frac{v_{\|}}{B}=\left.\frac{v_{\|}}{B}\right|_{0}-\frac{\tau}{\gamma p} \frac{\mathrm{D} p}{\mathrm{D} t}-\mathrm{v}_{\perp} \cdot \nabla \tau+\frac{\partial}{\partial \alpha} \int \frac{\partial \alpha}{\partial t} d \tau+\frac{\partial}{\partial \beta} \int \frac{\partial \beta}{\partial t} d \tau
$$

with

$$
\frac{\mathrm{D} p}{\mathrm{D} t}=\frac{\partial P(\alpha, \beta, t)}{\partial t}+\frac{\partial \Phi}{\partial \alpha} \frac{\partial P}{\partial \beta}-\frac{\partial \Phi}{\partial \beta} \frac{\partial P}{\partial \alpha}
$$

The term $v_{\|} /\left.B\right|_{0}$ in (54) and (55) represents a function of $\alpha, \beta$, and $t$ to be determined by boundary conditions.

The general solution for $\mathbf{E}$ and $\mathbf{v}$ for the given fields $\mathbf{B}(\mathbf{r}, t)$ and $p(\mathbf{r}, t)$ thus follows from (50), (51), and (54) with two free functions of $\alpha, \beta$, and $t$, following from prescribing the potential $\Phi$ and the parallel flow at one point on each field line. Such a boundary condition causes no obvious problem for lobe field lines that extend to large distances. For closed field lines, however, the ionosphere generally provides boundary conditions at both ends, which are not necessarily consistent with each other. Furthermore, boundary conditions for $\Phi$ are generally coupled to the magnetospheric field through the closure of magnetospheric field-aligned currents in the ionosphere. The inclusion of ionospheric boundary conditions thus restricts the possible field solutions in the magnetosphere. A basic approach to such a self-consistent closed chain of equations has been formulated by Vasyliunas [1970; see also Schindler and Birn, 1978]. A major piece of this chain, however, without the self-consistent magnetospheric magnetic field calculation, is the basis of the "Rice Convection Model" [Wolf, 1970, 1974; Harel and Wolf, 1976]. For the magnetotail part, only particle and entropy conservation have been included through a boundary condition for $v_{\|}$[Schindler and Birn. 1982; Birn and Schindler, 1983].

If north-sorth symmetry is imposed, then $v_{\|}=0$ at the equatorial plane, and (55) can be used directly to calculate $v_{\|}$at the ionosphere if the integrals are extended from the equatorial plane to the 
ionosphere. Using the average magnetospheric configuration, $\partial / \partial t=0$, and typical electric fields, Equation (55) implies an outflow into the ionosphere for earthward convection which by far exceeds the average particle precipitation. This is another way of formulating the "pressure inconsistency" [e.g., Erickson and Wolf, 1980; Schindler and Birn, 1986], which essentially states that the average observed magnetic field and pressure in the magnetosphere are inconsistent with a steady convection, as associated with typically observed ionospheric electric potentials, and the observed losses of particles from the closed field line region into the ionosphere, if adiabatic convection with $\gamma=5 / 3$ is assumed. The more common way of formulating this inconsistency is based on a different representation of (52), using mass conservation (1),

$$
\frac{\mathrm{D}}{\mathrm{D} t}\left(p V^{\gamma}\right)=0
$$

where $V$ represents the total volume of a magnetic flux tube of unit flux extending to the ionosphere,

$$
V=\int d \tau
$$

and flux tub ss are assumed to be closed at the ionosphere, i.e., outflow and inflow are neglected. In a steady state, Equation (57) implies that $p V^{\gamma}=$ const in each plane of convecting flux tubes. Using the average magnetic field, Equation (57) predicts a much larger pressure increase toward the Earth than is observed [Erickson and Wolf, 1980]. Relations between $p$ and $V$ (or, rather, the density $\rho$ ) that are derived from observation. give typically values of $\gamma$ less than $5 / 3$, even as low as $2 / 3$ [Birn and Schindler, 1982; Huang et al., 1989; Baumjohann and Paschmann, 1989]. We should add, however, that the statistically derived indices $\gamma$ may not be very meaningfull for properties of convection, if the scatter in the data, reflecting variations in the properties of plasma entering the plasma sheet, is larger than the typical variation of $p$ and $\rho$ due to the adiabatic or non-adiabatic convection across the region sampled.

Several possibilities exist to solve this apparent inconsistency: (1) While particle losses into the ionosphere do not seem to be sufficient to account for the discrepency for typical convection electric fields of $0.1-1 \mathrm{mV} / \mathrm{m}$, they may be sufficient to allow for steady convection when the electric field is smaller. (2) Kivelson and Spence [1988] (see also Spence and Kivelson, 1990) suggested that preferred losses of more energetic particles through the flanks of the magnetotail during earthward convection could account for the observed pressure deficiency; it is, however, not proved yet that this effect is quantitatively sufficient and that the observed particle distributions indeed show the reduction in high encrgy particles closer to the Earth, which corresponds to this mechanism. (3) If steady loss mechanisms are not sufficient, the 
conclusion must be that the average magnetosphere configuration is indeed not the result of a steady convection, but rather must include non-adiabatic dynamic processes, which lead to a loss of particles and/or energy [Erickson and Wolf, 1980; Schindler and Birn, 1982, 1986]. This conclusion leads to a plausible scenario for the development of magnetospheric substorms: Adiabatic convection, driven by the solar wind electric field which couples to the magnetosphere for southward IMF via frontside magnetic reconnection, deforms the magnetotail in a time-dependent way, governed by Equation (57), until a stability threshold is exceeded. The resulting instability of tearing type leads to the severance and tailward ejection of plasma and energy from the plasma sheet in the form of a plasmoid, as suggested, e.g;, by Hones [1977] and simulated by Birn and Hones [1981]. Schindler and Birn [1982] and Birn and Schindler [1983] showed for explicit sequences of equilibria, resulting from such forced convection, that they evolved toward instability, using criteria for current driven microinstabilities as well as for a collisionless ion tearing mode. A slightly different approach by Erickson [1984] and Hau et al. [1989], based on the attempt to find steady state solutions consistent with $p V^{5 / 3}=$ const at the expense of giving up consistency with the average magnetic field, indicates that, even if steady states are approached during the quasi-stacic evolution, they also would tend to lead to instability, because they develop a deep minimum of the equatorial $B_{z}$ near the inner edge of the plasma sheet, which would favor the onset of a tearing instability.

\section{SUMMARY AND DISCUSSIONS}

We have presented the most updated version of our general quasi-steady magnetotail theory. This version allows one to calculate general magnetotail configurations by solving ordinary differential equations and ordinary integrals with the free choice of boundary functions as in the general boundary value problem. The main assumptions are (1) isotropic pressure, (2) ideal MHD, (3) weak electric fields and slow temporal variations, measured by some small parameter $\delta$, and (4) the so-called "tail approximation," using the characteristic magnetotail property that spatial variations in $x$ and $y$ are smaller, by some factor $\epsilon$, than variations in $z$. The solutions that can be obtained by this theory are of order zero in the parameter $\delta$ but include the first order in $\epsilon$. For quasi-static cases, when the inertia terms are negligible, the first order terms in $\delta$ can also be found under certain conditions, as discussed in section 5. Particular solutions of the quasi-static equations were presented that include the following three-dimensional effects present in the actual magnetotail: Flaring of the tail in both $y$ and $z$, associated with the presence of $B_{z}$ and $B_{y}$; variation of the plasma sheet or current sheet thickness with $x$ and $y$, associated with a variation of $B_{z}$ with $x$ and 
$y$; field-aligned currents of region 1 type, which are found to be associated with the decrease of the tail flaring for larger distances; a net cross-tail magnetic field component $B_{y N}$, which may be chosen variable in $z$ at a given location. In addition, a particular steady model with field-aligned flow was presented, which indicates the important role of fast plasma flow for the transition of a thick closed plasma sheet to the more distant, much thinner current sheet and for the possibility of thick plasmoids moving in near equilibrium through the thin distant current sheet.

Refinements of the quasi-static theory which allow for pressure anisotropy are presently restricted to two-dimensional models [Bird and Beard, 1972; Cowley, 1978; Nötze! et al., 1985]. Such anisotropies are probably not important for the average large scale quiet magnetotail structure [Stiles et al., 1978; Nötzel et al., 1985], but they may play a role in the near-Earth region around $x \approx-10 R_{E}$, where thin current sheets may form in the substorm growth phase which seem to be associated with pressure anisotropy [Mitchell et al., 1990].

Another refinement of the present theory concerns the assumption of ideal MHD. This assumption is typically a good one for large scale magnetospheric structures as considered here [see, e.g., Vasyliunas, 1976]. It may break down, lowever, within localized regions, particularly at the reconnection sites in the near and far tail. While near-Earth reconnection is presumably part of a dynamic process, which is beyond our present approach, the distant reconnection process may occur in more steady fashion, which, in priciple, should be included in a quasi-steady theory. Our present approach does not incorporate non-ideal effects. It constitutes, however, the basis for an exterior solution to the reconnection problem, which has to be matched to an interior solution for the non-ideal "diffusion region" in a typical boundary layer approach to the reconnection problem [e.g., Petschek, 1964; Vasyliunas, 1975; Priest and Forbes, 1987]. 


\section{REFERENCES}

Baker, D. N., R. C. Anderson, R. D. Zwickl, and J. A. Slavin, Average plasma and magnetic field variations in the distant magnetotail associated with near-Earth substorm effects, J. Geophys. Res., 92, 71, 1987.

Baumjohann, W., and G. Paschmann, Determination of the polytropic index in the plasma sheet, $J$. Geophys. Res., 89, 295, 1989.

Birn, J., Self-consistent magnetotail theory: General solution for the qulet tail with vanishing field-aligned currents, J. Geophys. Res., 84, 5143, 1979.

Birn, J., Magnetotail equilibrium theory: The general three-dimensional solution, J. Geophys. Res., 9\&, $11,101,1987$.

Birn, J., Three-dimensional equilibria for the extended magnetotail and the generation of field-aligned current sheets, J. Geophys. Res., 94, 252, 1989.

Birn, J., The distortion of the magnetotail equilibrium structure by a net cross-tail magnetic field, $J$. Ceophys. Res., 95, 8019, 1990a.

Birn, J., Stretched three-dimensional plasma equilibria with field-aligned flow, Phys. Fluids B, in press, 1990b.

Birn, J., and E. W. Hones, Jr., Three-dimensional computer modeling of dynamic reconnection in the geomagnetic tail, J. Geophys. Res., 86, 6802, 1981.

Birn, J., and K. Schindler, Self-consistent theory of three-dimensional convection in the geomagnetic tail, J. Geophys. Res., S8, 6969, 1983.

Bird, M. K., and D. B. Beard, The selfconsistent geomagnetic tail under static conditions, J. Geophys. Res., 73, 907, 1968.

Cowley, S. W. H., The effect of pressure anisotropy on the equilibrium structure of magnetic current sheets, Planet. Space Sci., 26, 1037, 1978.

Eastman, T. E., and E. W. Hones, Jr., Chararteristics of the magnetospheric boundary layer and magnetopause layer as observed by Imp 6, J. Geophys. Res., 84, 2019, 1979.

Erickson, G. M., On the cause of X-line formation in the near-Earth plasma sheet: Results of adiabatic convection of plasma sheet plasma, in Magnetic Reconnection in Space and Laboratory Plasmas, edited by E. W. Ilones, Jr., Geophys. Monogr. Ser., vol. 30, p. 296, Am. Geophys. Union, Washington, D.C., 1984. 
Erickson, G. M., and R. A. Wolf, Is steady convection possible in the Earth's magnetotail?, Geophys. Res. Lett. 7, 897, 1980.

Fairfield, D. H., On the average configuration of the geomagnetic tail, J. Geophys. Res., 84, 1950, 1979.

Gosling, J. T., D. N. Baker, S. J. Bame, W. C. Feldman, and R. D. Zwickl, North-south and dawn-dusk plasma asymmetries in the distant tail lobes: ISEE 3, J. Geophys. Res., 90, 6354, 1985.

Cosling, J. T., M. F. 'Thomsen, S. J. Bame, and C. T. Russell, Accelerated plasma flows at the near-tail magnetopause, J. Gcophys. Res., 91, 3029, 1986.

Hardy, D. A., J. W. Freeman, and H. K. Hills, Plasma observations in the magnetotail, in Magnetospheric Particles and Fields, edited by B. M. McCormac, p. 89, D. Reidel, Hingham, Mass., 1976.

Harel, M., and R. A. Wolf, Convection, in Physics of Solar Planetary Environments, vol. 2, edited by D. J. Williams, p. 617, Am. Geophys. Union, Washington, D.C., 1976.

Hau, L.-N., R. A. Wolf, G.-H. Voigt, and C. C. Wu, Steady state magnetic field configurations for the Earth's magnetotail, J. Geophys. Pes., 94, 1303, 1989.

Hilmer, R. V., and G.-H. Voigt, The effects of a magnetic $B_{y}$ component on geomagnetic equilibria, $J$. Geophys. Res., 92, 8660, 1987.

Hones, E. W., Jr., Substorm processes in the magnetotail: Comments on "On hot tenuous plasmas, fireballs, and boundary layers in the Earth's magnetotail," by L. A. Frank, K. L. Ackerson, and R. P. Lepping, J. Geophys. Res., 82, 5633, 1977.

Hones, E. W., Jr., D. N. Baker, S. J. Bame, W. C. Feldman, J. T. Gosling, R. D. Zwickl, J. A. Slavin, E. J. Smith, and B. T. Tsurutani, Structure of the magnetotail at $220 R_{E}$ and its response to geomagnetic activity, Geophys. Res. Lett., 11, 5, 1984.

IIuang, C. Y., C. K. Goertz, L. A. Frank, and G. Rostoker, Observational determination of the adiabatic index in the quiet time plasma sheet, Geophys. Res. Lett., 16, 563, 1989.

Iijima, T., and T. A. Potemra, The amplitude distribution of field-aligned currents at northern high latitudes observed by Triad, J. Geophys, Res., 81, 2165, 1976.

Kivelson, M. G., and H. E. Spence, On the possibility of quasi-static convection in the quiet magnetotail, Geophys. Res. Lett., 15, 1541, 1988.

Lui, A. T. Y., Characteristics of the cross-tail current in the Earth's magnetotail, in Magnetospheric Currents, edited by T. A. Potemra, Geophys. Monogr. Ser., vol. 28, p. 158, Am. Geophys. Union, 
Washington, D.C., 1984.

Mitchell, D. G., D. J. Williams, C. Y. Huang, L. A. Frank, and C. T. Russell, Current carriers in the near-Earth cross-tail current sheet during substorm growth phase, Geophys. Res. Lett., 17, 583, 1990.

Nötzel, A., K. Schindler, and J. Birn, On the cause of appruximate pressure isotropy in the quiet near-Earth plasma sheet, J. Geophys, Rés., 90, 8293, 1985.

Papamastorakis, L., G. Paschmann, W. Baumjohann, B. U. Ö. Sonnerup, and Il. Lühr, Orientation, motion, and other properties of flux transfer event structures on September 4, 1984, J. Geophys. Res., 94, 8852, 1989.

Petschek, II. E., Magnetic field annihilation, NASA Spec.'Publ., SP-50, 425, 1964.

Priest, E. R., and T. G. Forbes, New models for fast steady state magnetic reconnection, J. Geophys. Res., $91,5579,1986$.

Rosenbauer, H., H. Grunwaldt, M. D. Montgomery, G. Paschmann, and N. Sckopke, Heos 2 plasma observations in the distant polar magnetosphere: The plasma mantle, J. Geophys. R\&s., 80, 2723, 1975.

Schindler, K., and J. Birn, Magnetospheric physics, Physics Reports, 47, 109, 1978.

Schindler, K., and J. Birn, Self-consistent theory of time-dependent convection in the Earth's magnetotail, J. Geophys. Res., 8\%, 2263, 1982.

Schindler, K., and J. Birn, Magnetotail theory, Space Sci. Rev., 44, 307, 1986.

Slavin, J. A., E. J. Smith, B. T. Tsurutani, D. G. Sibeck, II. G. Singer, D. N. Baker, J. T. Gosling, E. W. Hones, Jr, and F. L. Scarf, Substorm associated traveling compression regions in the distant tail: ISEE-3 geotail observations, Geophys. Res. Lett., 11, 657, 1984.

Slavin, J. A., E. J. Smith, D. G. Sibeck, D. N. Baker, R. D. Zwickl, and S.-I. Akasofu, An ISEE 3 study of average and substorm conditions in the distant magnetotail, J. Geophys. Res., 90, 10875, 1985.

Sonnerup, B. U. Ö., I. Papamastorakis, G. Paschmann, and H. Lühr, The magnetopause for large magnetic shear: Analysis of convection electric fields from AMPTE/IRM, J. Geophys. Res., 95, 10,541, 1990.

Spence, H. E., and M. G. Kivelson, The variation of the plasma sheet polytropic index along the midnight meridian in a finite width magnetotail, Geophys, Res. Lett., 17, 591, 1990.

Spence, H. E., M. G. Kivelson, and R. J. Walker, Static magnetic field models consistent with nearly isotropic plasma pressure, Geophys. Res. Lett., 14, 872, 1987.

Stiles, G. S., E. W. Hones, Jr., S. J. Bame, and J. R. Asbridge, Plasma sheet pressure anisotropies, J. 
Geophys, Res., 83, 3166, 1978.

Tsurutani, B. T., D. E. Jones, R. P. Lepping, E. J. Smith, and D. G. Sibeck, The relationship between the IMF $B_{y}$ and the distant tall $\left(150-238 R_{E}\right)$ lobe and plasmasheet $B_{y}$ flelds, Geophys. Res. Lett., 11, $1082,1984$.

Tsyganenko, N. A., Global quantitative inodels of the geomagnetic field in the cislunar magnetosphere for different disturbance levels, Planet. Space Sci., 35, 1347, 1987.

Tsyganenko, N. A, A magnetospheric fiold model with a warped tail current sheet, Planet. Space Sci., 37, $5,1989$.

Vasyliunas, V. M., in Particles and Fields in the Magnetosphere, edited by B. M. McCormac, p. 60, D. Reidel, Dordrecht, Holland, 1970.

Vasyliunas, V. M., Theoretical models of magnetic field line merging, 1, Rev. Geophys., 13, 303, 1975.

Vasyliunas, V. M., An overview of magnetospheric dynamics, in Magnetospheric Particles and Fields, edited by B. M. McCormac, p. 99, D. Reidel, Dordrecht, Ilolland, 1976.

Voigt, G.-H., and R. V. Hilmer, The influence of the IMF $B_{y}$ component on the Earth's magneto-hydrostatic magnetotail, in Magnetotail Physics, edited by A. T. Y. Lui, p. 91, Johns Iopkins University Press, Baltimore, MD, 1987.

Wolf, R. A., Effects of ionospheric conductivity on convective flow of plasma in the magnetosphere, $J$. Geophys. Res., 75, 4677, 1970.

Wolf, R. A., Calculations of magnetospheric electric fields, in Magnetospheric Physics, edited by B. M. McCormac, p. 167, D. Reidel, Ilingham, Mass., 1974.

Zwicki, R. D., D. N. Baker, S. J. Bame, W. C. Feldman, J. T. Gosling, E. W. Hones, Jr., D. J. McComas, B. T. Tsurutani, and J. A. Slavin, Evolution of the Earth's distant magnetotail: ISEE-3 electron plasma results, J. Geophys. Res., 89, 11007, 1984. 


\section{FIGURE CAPTIONS}

Fig. 1 Static magnetotall solution with $\hat{p}=\hat{p}(r)$, where $r^{2}=(x-a)^{2}+y^{2}$, and $B_{\phi}=0$ after Birn [1987]. Magnetic field lines are shown in three different, planes $\phi=$ const. The dashed lines represent the scale lenguh $L(x, y)$ for variations with $z$, equivalent to a current sheet half-thickness, and the shaded region indicates the region of closed field lines, that is, the plasma sheet.

Fig. 2 Perspective view of a magnetotail configuration after Birn [1989], showing a magnetic flux surface originating from a circle of $20 R_{E}$ radius at $x=-20 R_{E}$. For this configuration, the tall flaring decreases with distance from the Earth. The dashed line represents the characteristic scale length $L(x, y)$, and the shaded region indicates the closed plasma sheet region, which extends to infinity in this particular model.

Fig. 3 Distribution of the field-aligned current density at $x=-20 R_{E}$ for the configuration of Figure 2 . The top panel shows contours of constant $j_{\|}$with its magnitude increasing monotonically toward the separatrix from either side. The bottom panel shows a cut through the distribution of $j_{\|}$at $y=-15 R_{E}$. The values of $j_{\|}$are normalized by $j_{n}$, the peak cross-tail current density at $x=-20 R_{E}$. The dashed line indicates again the characteristic scale length $L$.

Fig. 4 Perspective view of a magnetotail configuration with a net cross-tail magnetic field component $B_{y N}$ after Birn [1990a]. As in Figure 2, the configuration is represented by a magnetic flux surface originating from a circle of $20 R_{E}$ radius at $x=-20 R_{E}$. The dashed lines indicate again the scale length $L$ and the shaded region the region of closed fleld lines with both ends inside of the $20 R_{E}$ circle at $x=-20 R_{E}$.

Fig. 5 Boundaries of the closed field line region in the equatorial plane after Birn [1990a] for various net cross-tail fields $b_{N}$, normalized by the typical lobe field strength. The dashed line represents the distant neutral line or separator, which defines the tailward boundary of the closed field line region for $b_{N}=0$. Note that the distribution of $B_{z}$ in the equatorial plane does not change significantly for the different cross-tail fields, and that $B_{z}$ is positive earthward of the dashed line in every case.

Fig. 6 Magnetic field lines of a two-dimensional magnetic island or plasmoid after Birn [1990b]. In the frame of the moving plasmoid these lines are also flow lines of the steady plasma flow around the plasmoid. The top panel represents the static case without flow, the two bottom panels cases with nearly Alfvénic flow $(m=0.995)$ around the plasmoid. The two top panels correspond to $20 \%$ pressure enhancement in the center of the plasmoid as compared to the pressure at $z=0$ for $|x| \rightarrow \infty$, while the bottom panel 
corresponds to $70 \%$ pressure enhancement. The horlzontal scales are normalized by an arbitrary length. $L_{x}$, the vertical scales on the left hand side by a characteristic length $L_{0}$, defined by tine current sheet half-thickness for $|x| \rightarrow \infty$ in the static case, and on the right hand side by the actual current sheet half-thickness $L$ in ean case. 


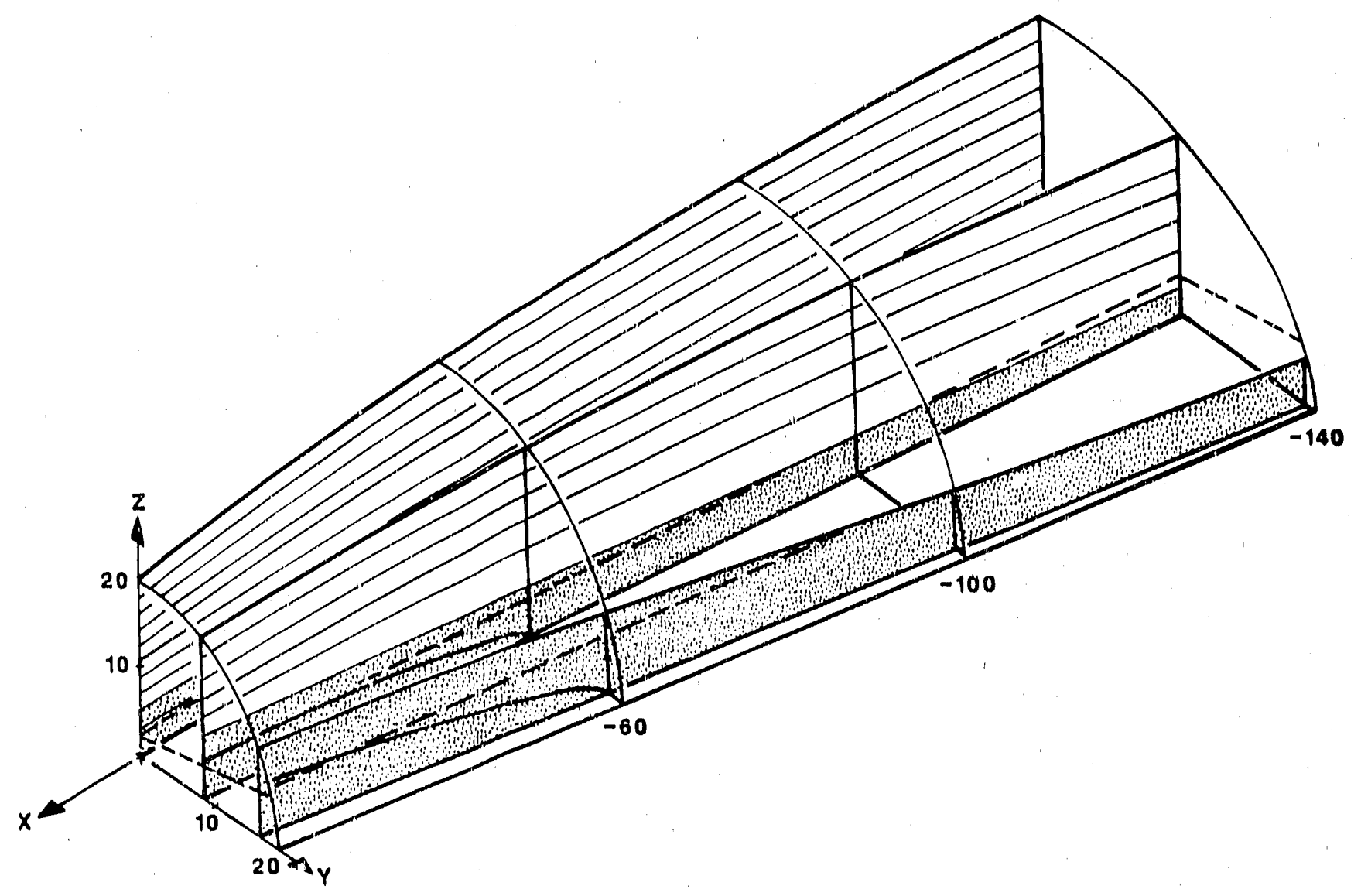

Fig. I 


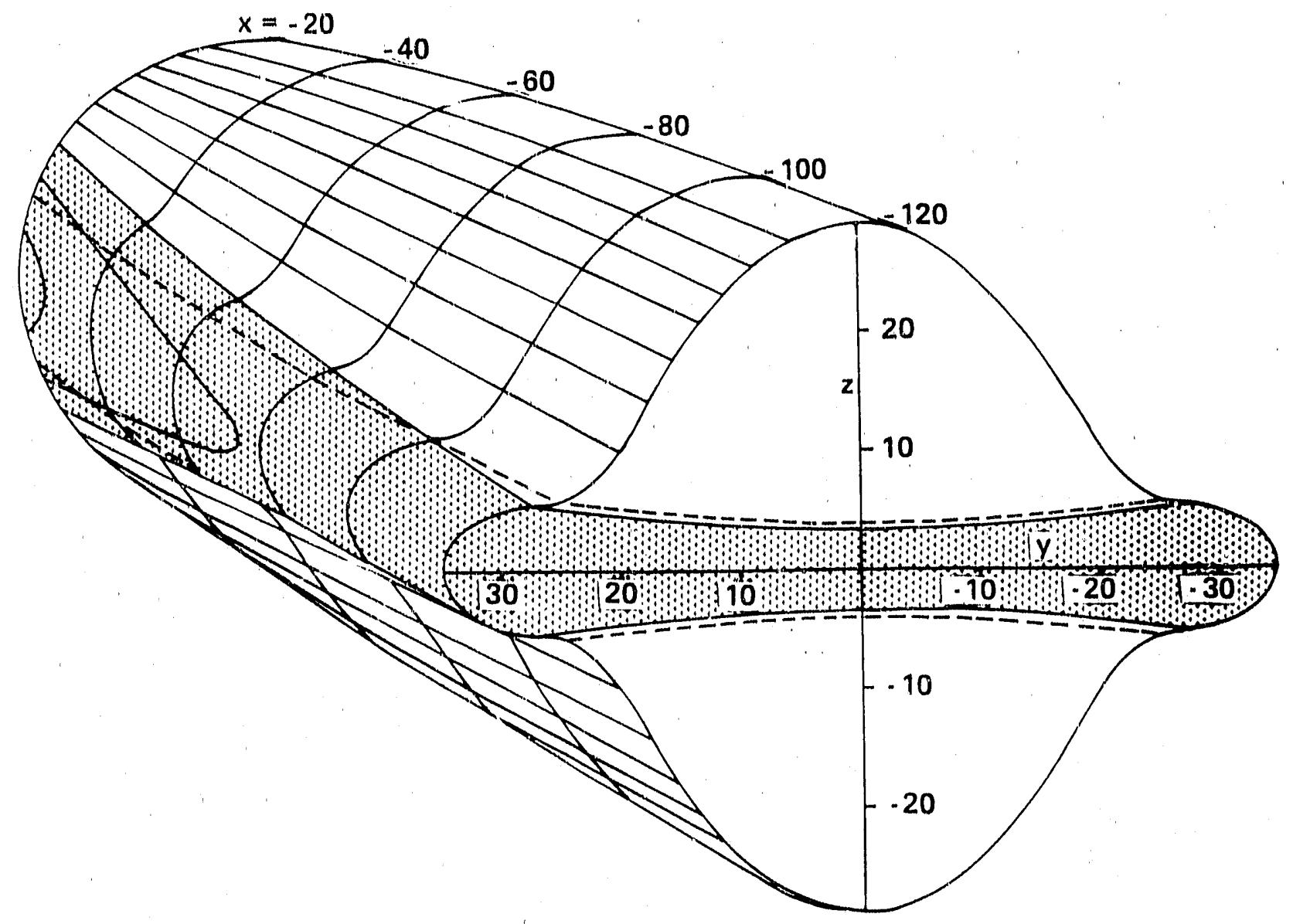

Fig. 2 

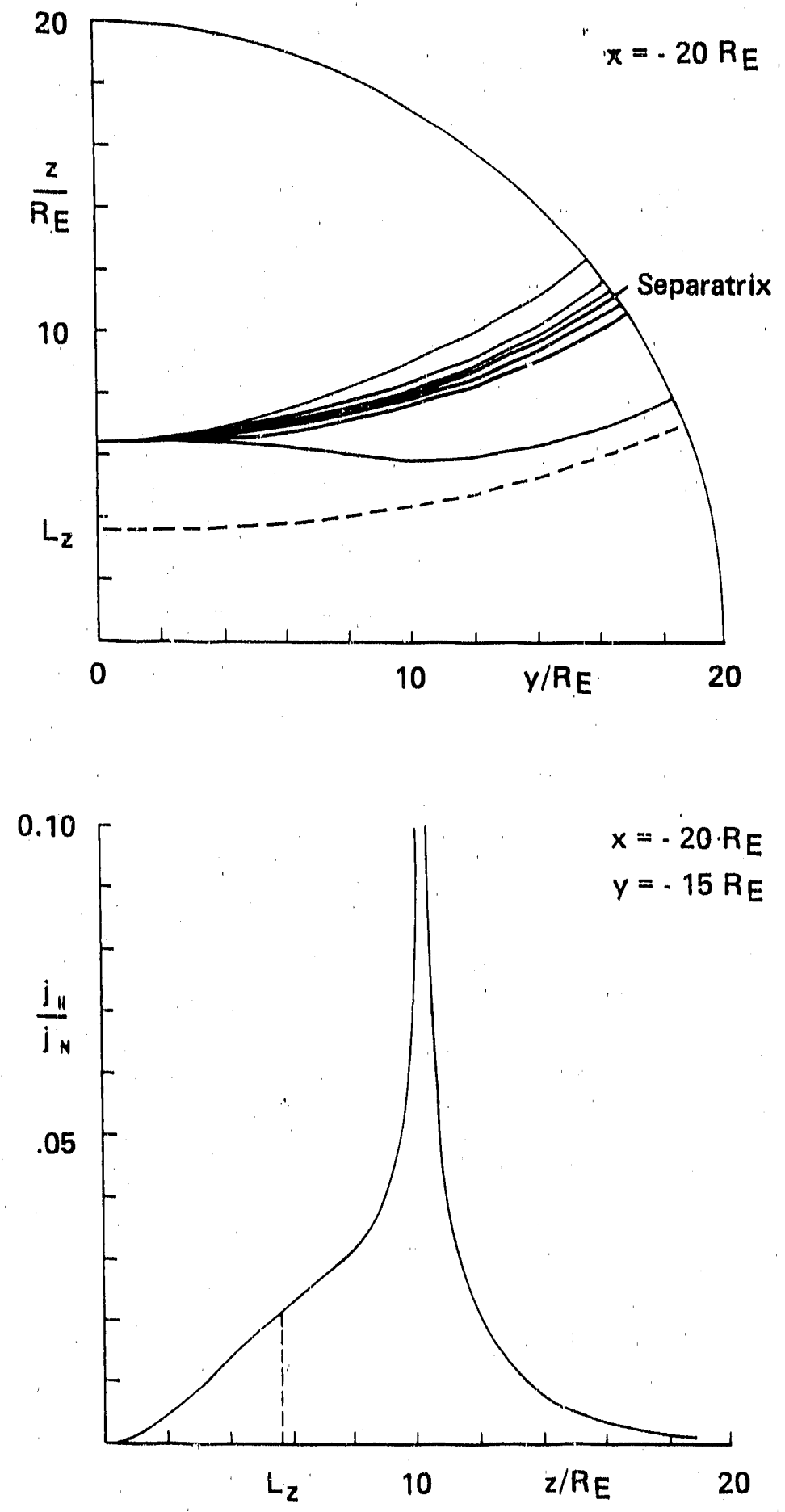

$F: g .3$ 


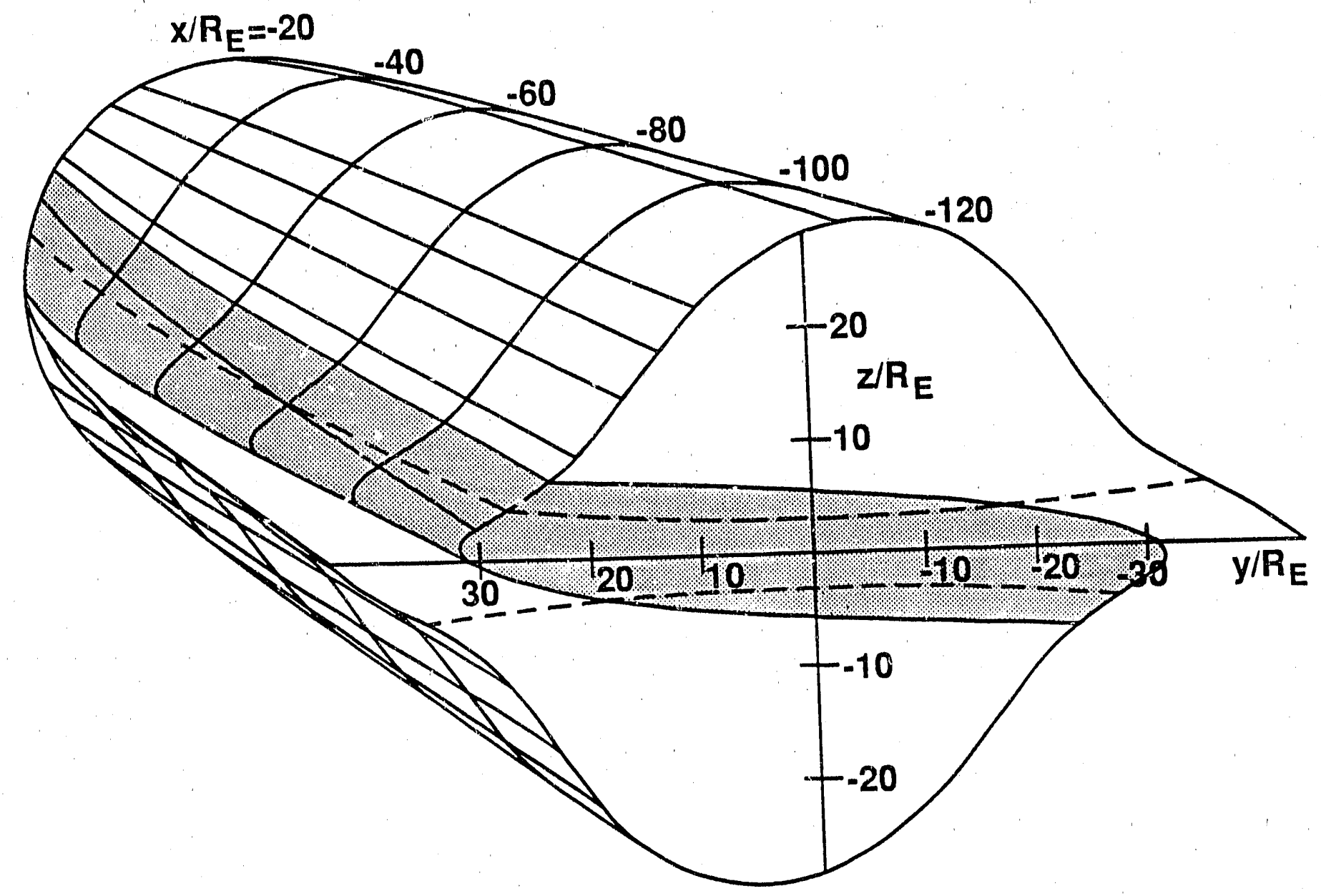

Fig. 4 


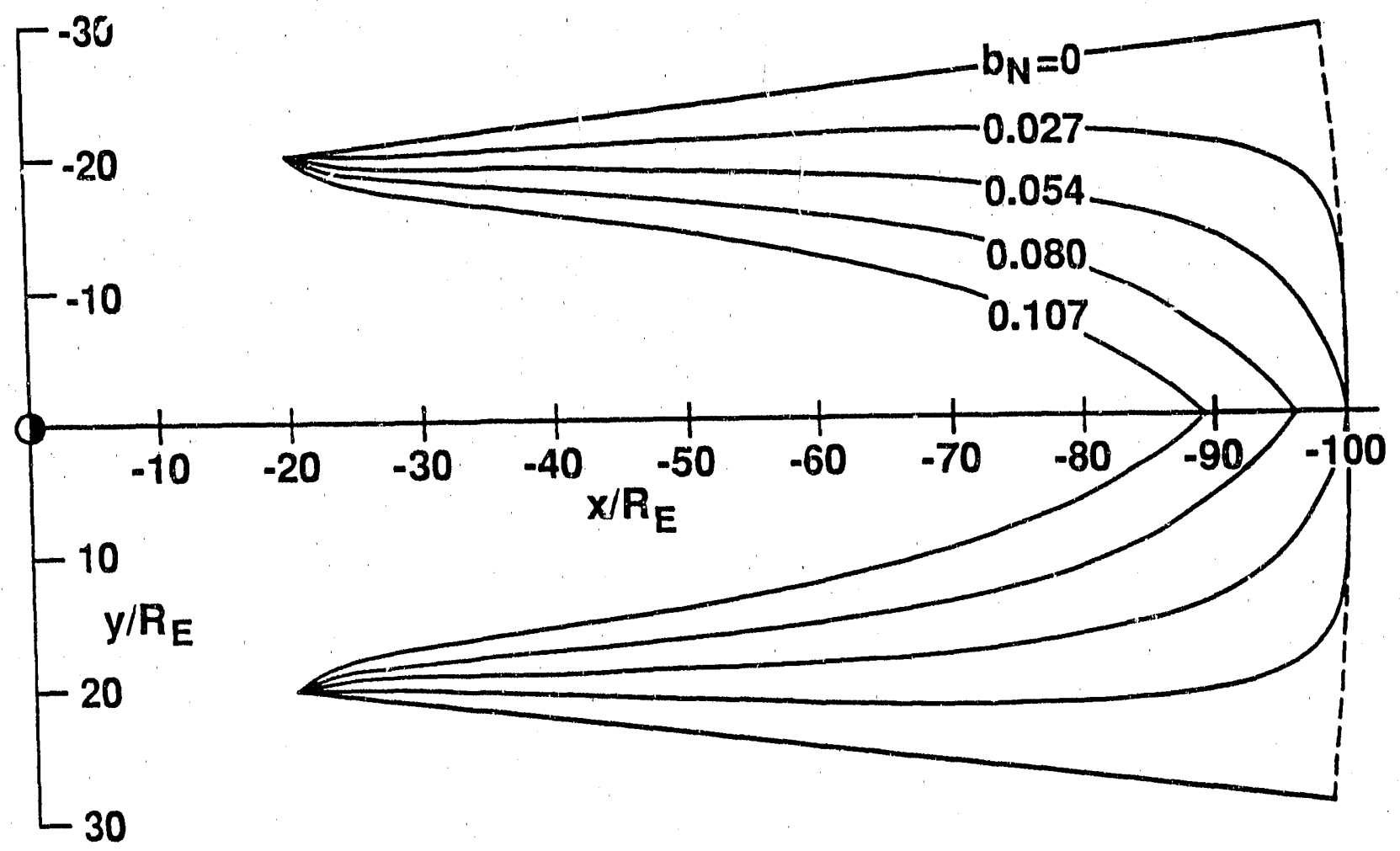

Fio. 5 

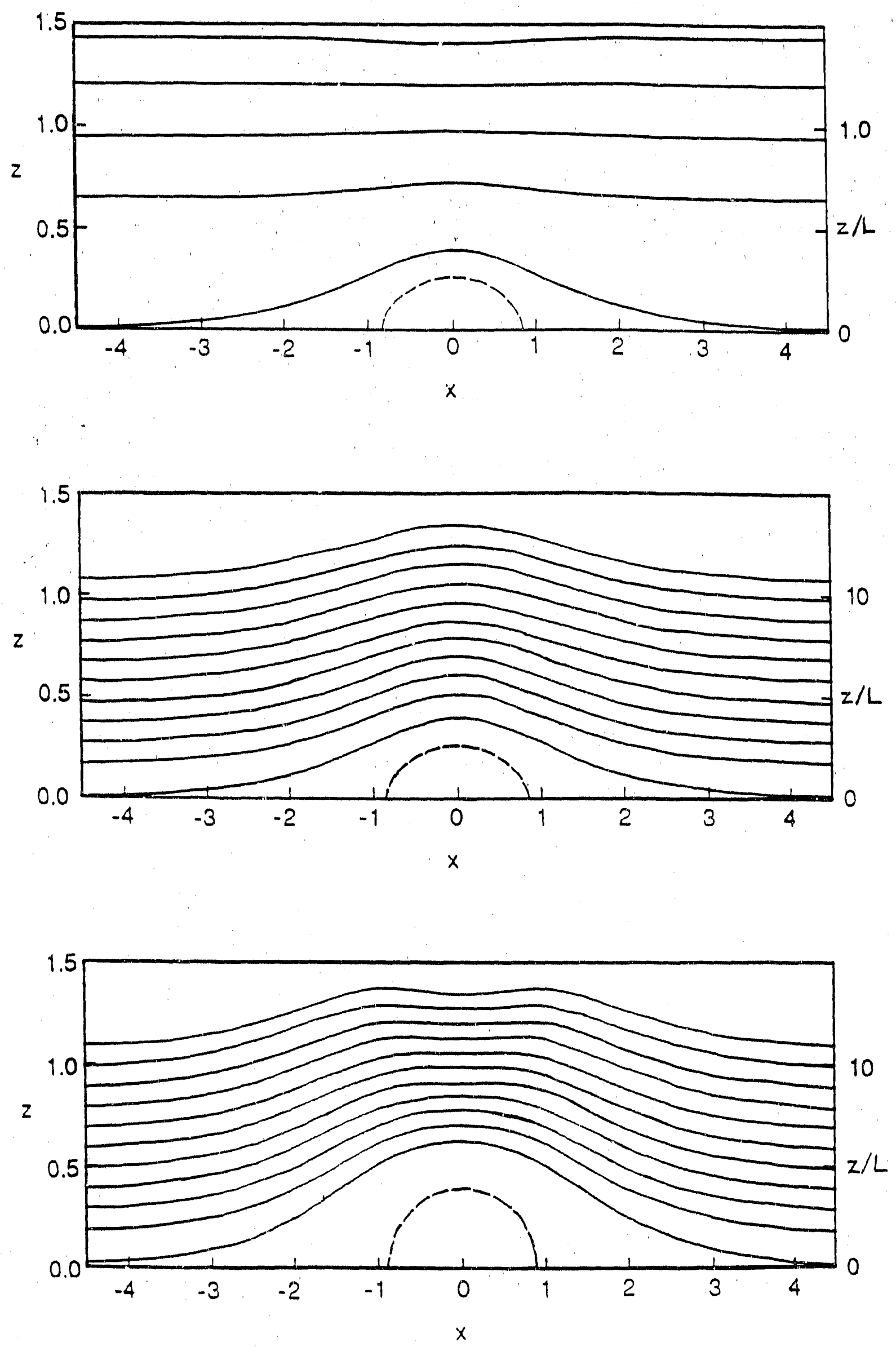

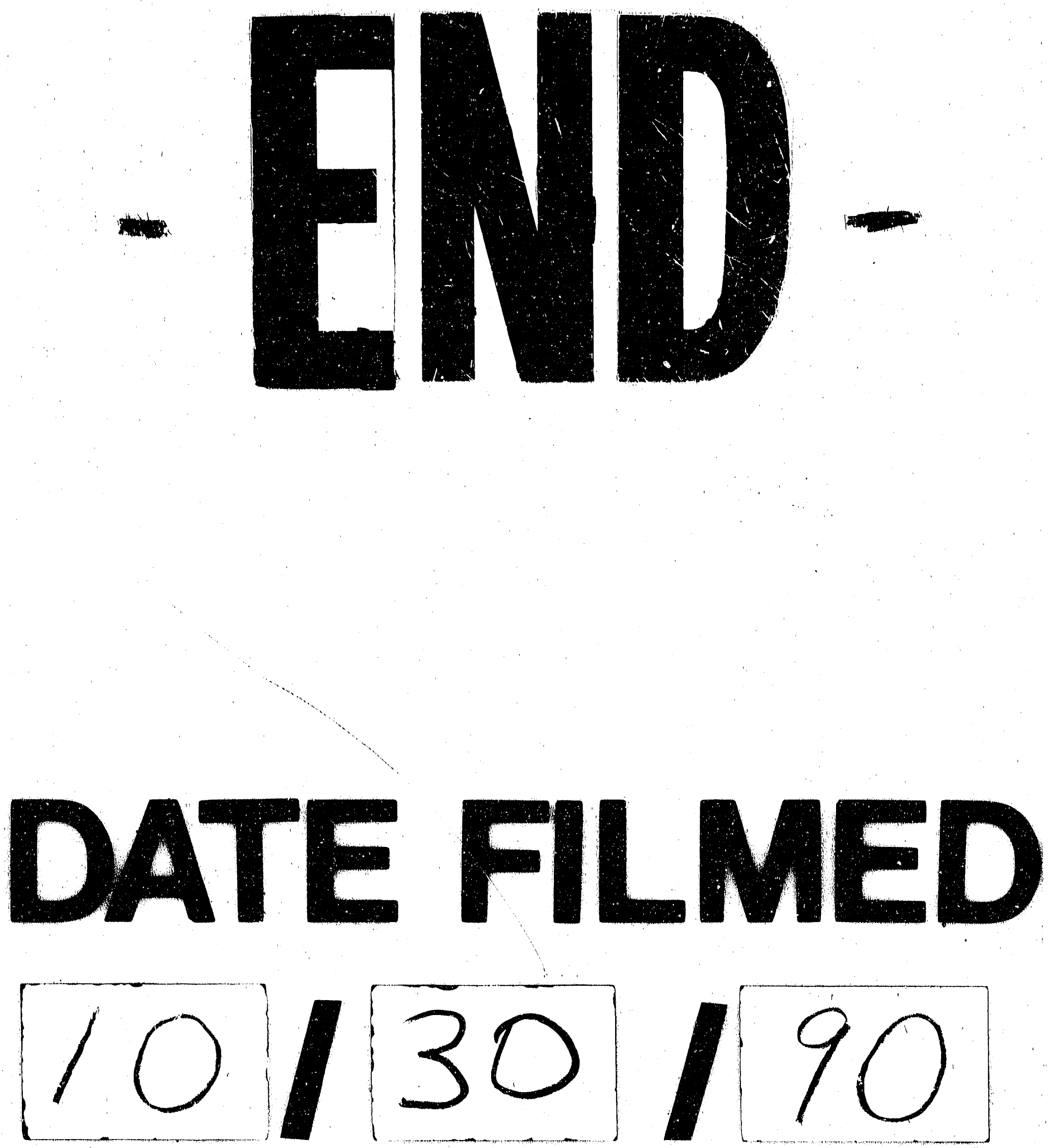


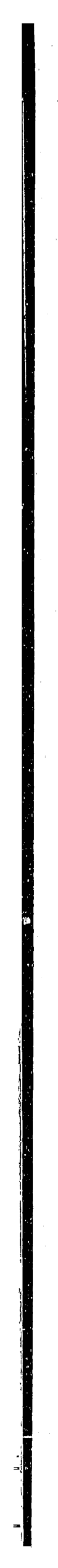

